\title{
Study on the Pretreatment of Poly (ether ether ketone) by Wet Chemistry and Its Effect on the Surface Morphology
}

\author{
Tong Zhai ${ }^{1, a}$, Lizhi Di ${ }^{2, b}$ and De'an Yang ${ }^{1, c}$ \\ ${ }^{1}$ School of Materials Science and Engineering, Tianjin University, Tianjin 300072, China; \\ 2 Tianjin Medical College, Tianjin 300222, China. \\ aztzhaitong@qq.com, bdilizh@126.com, cdayang@tju.edu.cn
}

Keywords: Poly (ether ether ketone), Wet Chemistry, Surface Morphology.

\begin{abstract}
Poly (ether ether ketone) was pretreated by wet chemistry and different surface morphology was obtained. In swelling treatment, with the increase of sulfuric acid concentration, the holes on surface changed from isolated structure to interpenetrated structure, the number and density of the holes increased, and the wall of the holes became thinner. Different kinds of cleaning solution could lead to different surface morphology. When sulfuric acid was used as cleaning solution, with the decrease of sulfuric acid concentration in cleaning, the holes on surface changed from isolated structure to interpenetrated structure, the number and density of the holes increased, and the wall of the holes became thinner.
\end{abstract}

\section{Introduction}

Poly(ether ether ketone) (PEEK) polymer a high performance semi-crystalline thermoplastic with outstanding features, such as superior mechanical properties, excellent thermal stability, low flammability, good radiation resistance, and excellent chemical and hydrolysis resistance.[1] Because of these properties, it is widely used in aircraft, aerospace, automobiles, energy industries, engineering applications, medicine and so on.

The surface treatment of PEEK is necessary and important when it used in some applications. The volume resistivity $\left(10^{14} \Omega \cdot \mathrm{m}\right)$ and surface resistance $\left(10^{15} \Omega\right)$ of PEEK are very high,[2] which made it be a good insulating material. However, it limits its use in the area of electronic conduction and electromagnetic interference shielding. Metallization of PEEK is a good approach to increase the electrical conductivity and electromagnetic interference shielding property.[3, 4] PEEK has excellent chemical stability with an inert smooth surface, which led to a weak adhesion between the PEEK and metal coating. So pretreatment of PEEK to obtain a rough and high-strength surface is important for the adhesion of metal coating. Mait et al.[5] chemically etched PEEK by $\mathrm{CrO}_{3}-\mathrm{H}_{2} \mathrm{SO}_{4}$ solution to increase the roughness and polarity of the surface, and then they metalized the PEEK surface by direct galvanic metallization with the help of cobalt sulfide. Zhai et al.[6] pretreated PEEK/MWCNT composite by $98 \mathrm{wt} \%$ concentrated sulfuric acid to generate a surface with interpenetrated holes, and then they metalized the PEEK/MWCNT composite by electroless plating method. PEEK has been used for medical implants due to its unique properties such as biocompatible, radiolucency, and mechanical properties similar to those of human bones.[7-9] In spite of these excellent attributes, the chemical and biological inertness of PEEK tends to limit bone fixation and deposition hydroxyapatite coating on PEEK which can enhance bone-implant integration. So the pretreatment of PEEK is important to improve the bone fixation. Almasi et al.[10] pretreated PEEK polymer by $98 \mathrm{wt} \%$ concentrated sulfuric acid and produced an interpenetrated holes surface, and then hydroxyapatite was deposited into the interpenetrated holes. In some industrial and medical areas, PEEK must be firmly adhered to PEEK or other materials to ensure device working normally. This also needs the pretreatment of PEEK. Awaja et al.[11] pretreated PEEK film by $\mathrm{CH}_{4} / \mathrm{O}_{2}$ radio-frequency plasma and two PEEK films were self-bonded together. The lap shear strength was 1.8 MPa for the samples with plasma treatment. However, the lap shear strength was only $0.3 \mathrm{MPa}$ for the samples without plasma 
treatment. Patrick et al.[12] pretreated PEEK polymer by $98 \mathrm{wt} \%$ concentrated sulfuric acid. The pretreated PEEK was bonded to epoxy resin with universal composite resin cement, the shear bond strength value was about $18 \mathrm{MPa}$ which was much higher than that without treatment. For PEEK-metal, PEEK-bone, PEEK-PEEK and so on, a good adhesion is necessary. The surface morphology is one of the most important factors for the adhesion. In this work, we studied the effect of wet chemistry treatment on the surface morphology of PEEK and obtained different surface morphology.

\section{Experimental Section}

\subsection{Materials}

PEEK polymer was supplied by Jiangsu Junhua High Performance Specialty Engineering Plastics (Peek) Products Co., Ltd. The dimension of the PEEK was about $6 \mathrm{~mm} \times 6 \mathrm{~mm} \times 3 \mathrm{~mm}$. All reagents used in this study were analytical reagent grade.

\section{2 Methods and Characterization}

First, the PEEK samples were degreased in alkaline solution $\left(\mathrm{NaOH} 25 \mathrm{~g} / \mathrm{L}, \mathrm{Na}_{2} \mathrm{CO}_{3} 40 \mathrm{~g} / \mathrm{L}\right.$, $\mathrm{Na}_{3} \mathrm{PO}_{4} 35 \mathrm{~g} / \mathrm{L}$, emulsifier OP-10 $2 \mathrm{~mL} / \mathrm{L}$ ) at $60{ }^{\circ} \mathrm{C}$ for $15 \mathrm{~min}$. The purpose of degreasing is to remove the oil stain on the surface so that the uniformity of the swelling process can be improved. After taking out of the degreasing solution, the samples were rinsed with water and dried. PEEK has excellent chemical and solvent resistance and is insoluble in most common solvents, with the exception of some strong proton acids. In this study, sulfuric acid $\left(\mathrm{H}_{2} \mathrm{SO}_{4}\right)$ was used as swelling agent. The dried PEEK samples were put into sulfuric acid with different concentration for 30s at room temperature. After taking out of the swelling agent, the samples were put into cleaning solution (water, ethanol absolute, dimethyl sulfoxide and sulfuric acid with different concentration) for 10min. After taking out of the cleaning solution, the samples were rinsed with water and dried. The surface morphology of PEEK samples with different treatment were observed by scanning electron microscopy (SEM, Hitachi S4800).

\section{Results and Discussion}

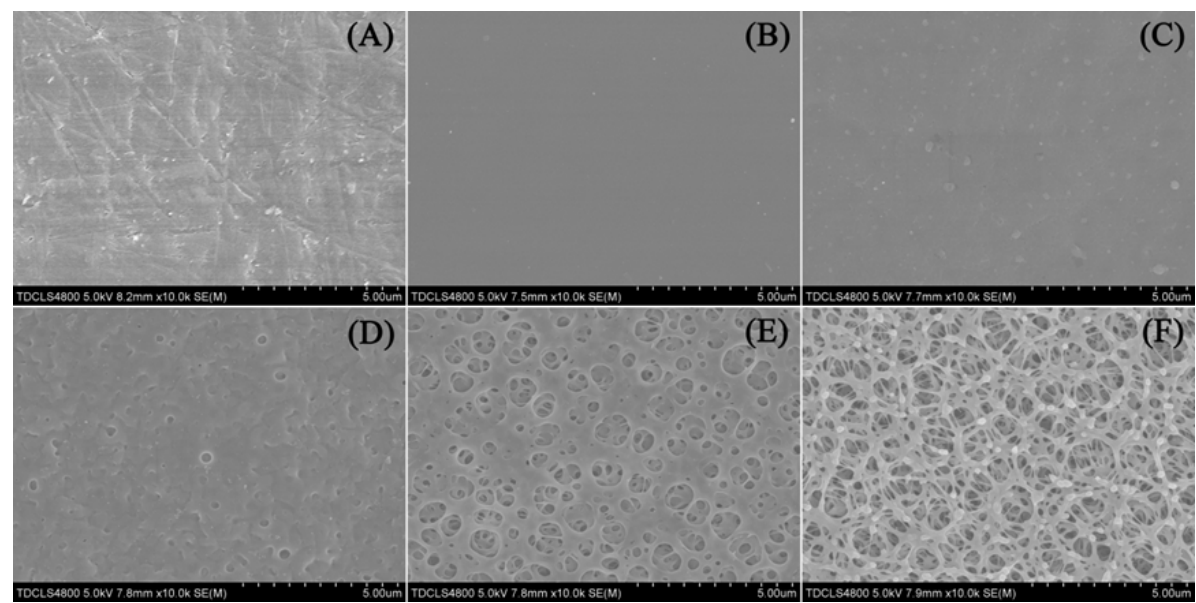

Figure 1 SEM images of the PEEK samples before swelling (A) and after swelling in $83.3 \mathrm{v} / \mathrm{v} \%(\mathrm{~B})$, $88.2 \mathrm{v} / \mathrm{v} \%(\mathrm{C}), 93.8 \mathrm{v} / \mathrm{v} \%(\mathrm{D}), 96.8 \mathrm{v} / \mathrm{v} \%(\mathrm{E}), 100 \mathrm{v} / \mathrm{v} \%(\mathrm{~F}) \mathrm{H}_{2} \mathrm{SO}_{4}$ aqueous solution.

Figure 1 (A) shows the surface morphology of the PEEK after degreasing treatment. The surface was smooth and compact before swelling. No holes or flaws could be seen on the surface, and only some traces of mold were left on the surface. After degreasing and drying, the PEEK samples were swelled in $\mathrm{H}_{2} \mathrm{SO}_{4}$ aqueous solution with different concentration (volume fraction: 83.3, 88.2, 93.8, 96.8, and $100 \mathrm{v} / \mathrm{v} \%$ ) for 30s at room temperature. Then the samples were put into water at room temperature. After the samples were swelled by $83.3 \mathrm{v} / \mathrm{v} \% \mathrm{H}_{2} \mathrm{SO}_{4}$, as shown in Figure 1(B), the traces of mold were disappeared which indicated the PEEK on surface was dissolved. And the surface became more smooth and compact, which had a negative impact on adhesion. When the volume 
fraction of $\mathrm{H}_{2} \mathrm{SO}_{4}$ increased to $88.2 \mathrm{v} / \mathrm{v} \%$, as shown in Figure 1(C), the surface morphology was similar to that in Figure 1(B). When the volume fraction of $\mathrm{H}_{2} \mathrm{SO}_{4}$ was $93.8 \mathrm{v} / \mathrm{v} \%$, a few isolated holes at nano scale were appeared on the surface (Figure 1(D)). However, the number of the holes was small and the depth of the holes was shallow. When the volume fraction of $\mathrm{H}_{2} \mathrm{SO}_{4}$ reached $96.8 \mathrm{v} / \mathrm{v} \%$, a lot of interpenetrated holes at micron scale were appeared on the surface (Figure 1(E)). When the volume fraction of $\mathrm{H}_{2} \mathrm{SO}_{4}$ was $100 \mathrm{v} / \mathrm{v} \%$, the surface also showed an interpenetrated holes structure (Figure 1(F)). However, compared with Figure 1(E), the number and density of the holes increased, and the wall of the holes became thinner. The holes surface morphology is important for the adhesion of PEEK-metal, PEEK-bone, PEEK-PEEK and so on, because it increased the surface area of PEEK and provided the mechanical bonding sites between PEEK and other materials (known as anchorage effect). From Figure 1, we found the surface morphology was very sensitive to $\mathrm{H}_{2} \mathrm{SO}_{4}$ concentration. And only when the volume fraction of $\mathrm{H}_{2} \mathrm{SO}_{4}$ was higher than $93.8 \mathrm{v} / \mathrm{v} \%$, a holes surface could be produced. On this basis, with the increase of sulfuric acid concentration, the holes on surface changed from isolated structure to interpenetrated structure, the number and density of the holes increased, and the wall of the holes became thinner.

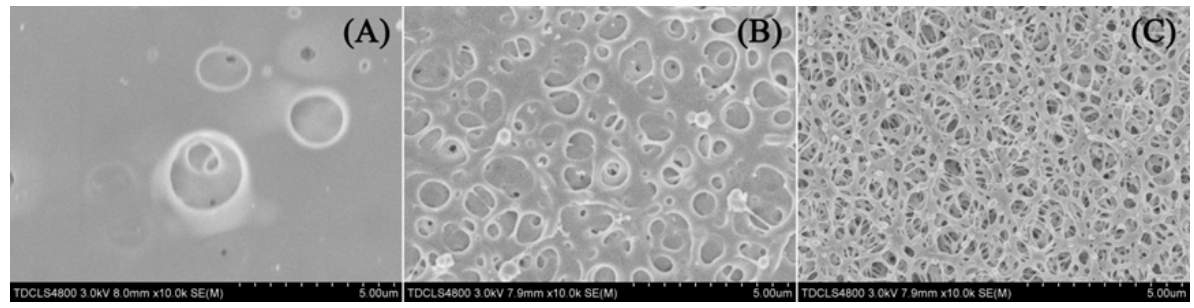

Figure 2 SEM images of the PEEK samples swelling in $100 \mathrm{v} / \mathrm{v} \% \mathrm{H}_{2} \mathrm{SO}_{4}$ and cleaning in 83.0 $\mathrm{v} / \mathrm{v} \%(\mathrm{~A}), 63.5 \mathrm{v} / \mathrm{v} \%(\mathrm{~B}), 37.3 \mathrm{v} / \mathrm{v} \%(\mathrm{C}) \mathrm{H}_{2} \mathrm{SO}_{4}$ aqueous solution.

After degreasing and drying, the PEEK samples were swelled in $100 \mathrm{v} / \mathrm{v} \% \mathrm{H}_{2} \mathrm{SO}_{4}$ for $30 \mathrm{~s}$ at room temperature. Then the samples were put into $\mathrm{H}_{2} \mathrm{SO}_{4}$ aqueous solution with different concentration (83.0, 63.5 and $37.3 \mathrm{v} / \mathrm{v} \%$ ) for 10min at room temperature. Then the samples were rinsed with water and dried. When the cleaning solution was $83.0 \mathrm{v} / \mathrm{v} \% \mathrm{H}_{2} \mathrm{SO}_{4}$, the surface showed a small amount of isolated holes at micron scale (Figure 2(A)). However, when the cleaning solution was $63.5 \mathrm{v} / \mathrm{v} \%$ $\mathrm{H}_{2} \mathrm{SO}_{4}$, the surface showed an interpenetrated holes structure (Figure 2(B)). Compared with that cleaned in waster (Figure 1(F)), the density of the holes decreased, and the wall of the holes was thicker. When the cleaning solution was $37.3 \mathrm{v} / \mathrm{v} \% \mathrm{H}_{2} \mathrm{SO}_{4}$, the surface also showed an interpenetrated holes structure (Figure 2(C)). The wall of the holes was thinner than that clean with $63.5 \mathrm{v} / \mathrm{v} \% \mathrm{H}_{2} \mathrm{SO}_{4}$, and it was thicker than that clean with water. So when $\mathrm{H}_{2} \mathrm{SO}_{4}$ was used as cleaning solution, with decreasing the concentration of $\mathrm{H}_{2} \mathrm{SO}_{4}$, the holes on surface changed from isolated structure to interpenetrated structure, the number and density of the holes increased, and the wall of the holes became thinner.

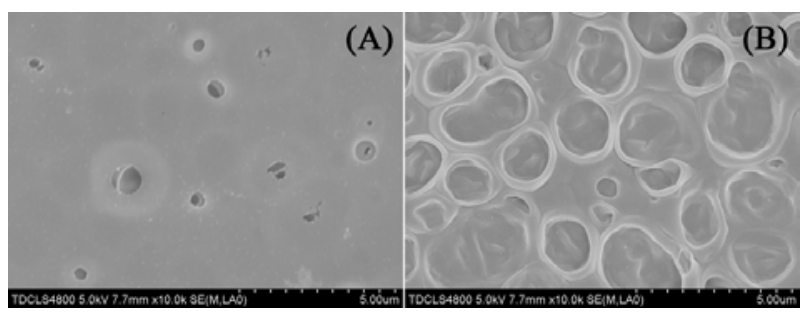

Figure 3 SEM images of the PEEK samples swelling in $100 \mathrm{v} / \mathrm{v} \% \mathrm{H}_{2} \mathrm{SO}_{4}$ and cleaning in ethanol absolute (A) and dimethyl sulfoxide (B).

After degreasing and drying, the PEEK samples were swelled in $100 \mathrm{v} / \mathrm{v} \% \mathrm{H}_{2} \mathrm{SO}_{4}$ for $30 \mathrm{~s}$ at room temperature. Then the samples were put into ethanol absolute and dimethyl sulfoxide for 10min at room temperature. Then the samples were rinsed with water and dried. When the PEEK was cleaned in ethanol absolute, the surface showed a small amount of isolated holes at micron scale (Figure 3(A)). However, when the PEEK was cleaned in dimethyl sulfoxide, the surface showed a lot of open holes structure (Figure 3(B)). The size of the holes was larger than that cleaned in water (Figure 1(F)) and ethanol absolute (Figure 3(A)). Different kinds of cleaning solution could lead to different surface 
morphology.

\section{Conclusions}

We studied the effects of sulfuric acid concentration in swelling treatment, the kind and concentration of cleaning solution on the surface morphology of PEEK. Through controlling those influential factors, the PEEK with different surface morphology was obtained. This is helpful for further studying the combination of PEEK with metal coating, bone, resin and other materials.

\section{References}

[1] A.M. Diez-Pascual, M. Naffakh, C. Marco, G. Ellis, M.A. Gomez-Fatou, High-performance nanocomposites based on polyetherketones, Prog. Mater. Sci., 57 (2012) 1106-1190.

[2] H.S. Wang, G.B. Wang, W.L. Li, Q.T. Wang, W. Wei, Z.H. Jiang, S.L. Zhang, A material with high electromagnetic radiation shielding effectiveness fabricated using multi-walled carbon nanotubes wrapped with poly(ether sulfone) in a poly(ether ether ketone) matrix, J. Mater. Chem., 22 (2012) 21232-21237.

[3] T. Zhai, L. Di, D.a. Yang, Electroless nickel-phosphorus coating on poly (ether ether ketone)/carbon nanotubes composite, Electronic Materials Letters, 10 (2014) 631-636.

[4] B. Zhou, L. Kong, L. Niu, J. Lian, G. Li, Effects of passivation on the properties of Ni-P alloy coating deposited on CFs reinforced PEEK, Surface and Coatings Technology, 232 (2013) 269-274.

[5] T. Mai, J. Schultze, G. Staikov, Relation between surface preconditioning and metal deposition in direct galvanic metallization of insulating surfaces, Journal of Solid State Electrochemistry, 8 (2004) 201-208.

[6] T. Zhai, B. Liu, C.-H. Ding, L.-X. Lu, C. Zhang, K.-G. Xue, D.-A. Yang, Ni-P electroless deposition directly induced by sodium borohydride at interconnected pores of poly (ether ether ketone)/multiwalled carbon nanotubes composites surface, Surface and Coatings Technology, 272 (2015) 141-148.

[7] W. Kaczorowski, D. Batory, W. Szymanski, P. Niedzielski, Evaluation of the surface properties of PEEK substrate after two-step plasma modification: Etching and deposition of DLC coatings, Surface and Coatings Technology, 265 (2015) 92-98.

[8] Y. Zhao, H.M. Wong, W. Wang, P. Li, Z. Xu, E.Y. Chong, C.H. Yan, K.W. Yeung, P.K. Chu, Cytocompatibility, osseointegration, and bioactivity of three-dimensional porous and nanostructured network on polyetheretherketone, Biomaterials, 34 (2013) 9264-9277.

[9] H. Zhou, V.K. Goel, S.B. Bhaduri, A fast route to modify biopolymer surface: A study on polyetheretherketone (PEEK), Mater. Lett., 125 (2014) 96-98.

[10] D. Almasi, S. Izman, M. Assadian, M. Ghanbari, M.A. Kadir, Crystalline ha coating on peek via chemical deposition, Appl. Surf. Sci., 314 (2014) 1034-1040.

[11] F. Awaja, S. Zhang, N. James, D.R. McKenzie, Enhanced autohesive bonding of polyetheretherketone (PEEK) for biomedical applications using a methane/oxygen plasma treatment, Plasma Processes and Polymers, 7 (2010) 1010-1021.

[12] P.R. Schmidlin, B. Stawarczyk, M. Wieland, T. Attin, C.H. Hämmerle, J. Fischer, Effect of different surface pre-treatments and luting materials on shear bond strength to PEEK, dental materials, 26 (2010) 553-559. 